\title{
Quarkmasses and the strong coupling from recent $R(s)$ measurements
}

\section{Matthias Steinhauser*}

Institut für Theoretische Teilchenphysik, Universität Karlsruhe, D-76128 Karlsruhe, Germany

E-mail: matthias.steinhauser@uka.de

In this contribution we describe the determination of the charm and bottom quark masses and the strong coupling constant from a combination of recent experimental data for the total hadronic cross section and precise perturbative predictions within QCD. The results read $m_{c}\left(m_{c}\right)=1.286(13) \mathrm{GeV}, m_{b}\left(m_{b}\right)=4.164(25) \mathrm{GeV}$ and $\alpha_{s}^{(5)}\left(M_{Z}^{2}\right)=0.119_{-0.011}^{+0.009}$.

8th International Symposium on Radiative Corrections

October 1-5, 2007

Florence, Italy

${ }^{*}$ Speaker. 


\section{Introduction}

The strong coupling constant and the masses of the six quarks constitute the fundamental parameters of QCD which is a strong motivation to determine their values with highest possible precision. Precise quark masses are furthermore important in the context of spectroscopy, $B$ meson and Higgs boson decays and Yukawa unification in Grand Unified Theories.

In this contribution we describe the extraction of $\alpha_{s}$ (Section 2) and the charm and bottom quark masses (Section 3) from recent measurements of the total hadronic cross section in $e^{+} e^{-}$ annihilation in combination with recent precision calculations.

\section{Strong coupling constant}

Recently the CLEO collaboration has published a measurement of $R(s)$ for seven different center-of-mass values $\sqrt{s}$ in the range between $7 \mathrm{GeV}$ and $10 \mathrm{GeV}$ [1]. The uncertainty amounts to about $2 \%$ and it is thus tempting to compare these measurements with the theoretical predictions for $R(s)$ given by

$$
R(s)=\frac{\sigma\left(e^{+} e^{-} \rightarrow \text { hadrons }\right)}{\sigma_{\mathrm{pt}}},
$$

where $\sigma_{\mathrm{pt}}=4 \pi \alpha^{2} /(3 s) . R(s)$ is known to order $\alpha_{s}^{2}$ including the complete mass dependence and at order $\alpha_{s}^{3}$ mass corrections up to the quartic order are available (see Refs. [2, 3, 4] for recent compilations). All available corrections are included in the program rhad [4], which has been used for the present analysis.

In a first step we evolve the seven extracted values for $\alpha_{s}$ to a common energy scale which we choose to be $9 \mathrm{GeV}$. We obtain $\alpha_{s}^{(4)}\left(9^{2} \mathrm{GeV}^{2}\right)=0.160 \pm 0.024 \pm 0.024$, where the first error combines statistical and uncorrelated systematic uncertainties and the second one gives the correlated systematic error. The superscript "(4)" indicates that at this point we are still working in a version of QCD where only the four lighter quark flavours are active. The transition to five active quarks is achieved with the help of a proper matching when crossing the flavour threshold. It is implemented in convenient form in the Mathematica package RunDec [5] which is based on the works [6, 7]. Using the proper matching and running of the strong coupling from $9 \mathrm{GeV}$ to $M_{Z}$ we thus obtain from $\alpha_{s}^{(4)}\left(9^{2} \mathrm{GeV}^{2}\right)$

$$
\alpha_{s}^{(5)}\left(M_{Z}^{2}\right)=0.110_{-0.012-0.011}^{+0.010+0.010}=0.110_{-0.017}^{+0.014}
$$

where after the second equality sign the uncertainties have been combined in quadrature.

The central value in Eq. (2.2) differs by one standard deviation from the one of Ref. [1], $\left.\alpha_{s}^{(5)}\left(M_{Z}^{2}\right)\right|_{\text {CLEO }}=0.126 \pm 0.005_{-0.011}^{+0.015}$. This is explained by the missing charm mass effects in the theory predictions for $R(s)$ and the missing matching corrections at the bottom threshold in Ref. [1].

It is instructive to combine the result from Eq. (2.2) with the $\alpha_{s}$ values obtained in Ref. [8], $\alpha_{s}^{(4)}\left(5^{2} \mathrm{GeV}^{2}\right)=0.235_{-0.047}^{+0.047}$ and $\alpha_{s}^{(5)}\left(M_{Z}^{2}\right)=0.124_{-0.014}^{+0.011}$, which was based on earlier measurements by BES [9], MD-1 [10] and CLEO [11]. Adding the correlated and uncorrelated errors of the different experiments in quadrature, the final result $\alpha_{s}^{(4)}\left(9^{2} \mathrm{GeV}^{2}\right)=0.182_{-0.025}^{+0.022}$ represents the combined information on the strong coupling from these $R$ measurements in the region below the 
bottom threshold and corresponds to $\alpha_{s}^{(5)}\left(M_{Z}^{2}\right)=0.119_{-0.011}^{+0.009}$. Although the uncertainties are still slightly larger the extraction of $\alpha_{s}$ from the inclusice quantity $R(s)$ becomes competitive with the results entering the world average (see, e.g., $[12,13])$.

\section{Charm and Bottom quark mass}

The method described in this contribution goes back to 1977 [14] and was applied to next-tonext-to-leading order (NNLO) in Ref. [8]. The NNNLO analysis, including updated experimental input, was presented in Ref. [15].

The basic object which enters our analysis is the photon polarization function defined through

$$
\left(-q^{2} g_{\mu v}+q_{\mu} q_{v}\right) \Pi\left(q^{2}\right)=i \int \mathrm{d} x e^{i q x}\left\langle 0\left|T j_{\mu}(x) j_{v}^{\dagger}(0)\right| 0\right\rangle,
$$

with $j_{\mu}$ being the electromagnetic current. The normalized total cross section for hadron production in $e^{+} e^{-}$annihilation is then given by $R(s)=12 \pi \operatorname{Im}\left[\Pi\left(q^{2}=s+i \varepsilon\right)\right]$. In the following we add a subscript $Q$ to indicate the contribution from the heavy quark $Q$.

The idea for extracting a quark mass value $m_{Q}$ is based on moments constructed from $\Pi_{Q}$. On one hand one can compute the Taylor expansion of $\Pi_{Q}\left(q^{2}\right)$ around $q^{2}=0$ and obtain the so-called "theory-moments" from

$$
\mathscr{M}_{n}^{\mathrm{th}}=\left.\frac{12 \pi^{2}}{n !}\left(\frac{\mathrm{d}}{\mathrm{d} q^{2}}\right)^{n} \Pi_{Q}\left(q^{2}\right)\right|_{q^{2}=0} .
$$

The three-loop contribution to $\Pi_{Q}\left(q^{2}\right)$ up to $n=8$ within QCD has been computed in Refs. [16, 17, 18] and the four-loop calculation for $n=1$ has been performed in Refs. [19, 20]. In the analysis of Ref. [15] also two-loop QED corrections and non-perturbative contributions have been considered. The former are quite small and the latter show a visible effect only in the case of the charm quark.

From dimensional considerations we have $m_{Q} \sim\left(\mathscr{M}_{n}^{\text {th }}\right)^{\frac{1}{2 n}}$ which implies a stronger dependence of $m_{Q}$ on variations of $\mathscr{M}_{n}^{\text {th }}$ for smaller values of $n$. Furthermore, higher values of $n$ require a careful theoretical treatment of the threshold region and the construction of an effective theory. The analysis performed in Ref. [15] is restricted to $n=1,2,3$ and 4. Note that precise mass values can only be obtained for the three lowest moments since the non-perturbative contributions become too big already for $n=4$.

One of the major advantages of the method discussed in this paper is that we can adopt the $\overline{\mathrm{MS}}$ scheme for the quark mass entering $\Pi_{Q}\left(q^{2}\right)$ in Eq. (3.2) and thus directly extract the corresponding value for the mass.

In order to extract experimental moments one exploits the analyticity of $\Pi_{Q}$ and arrives at the experimental moment given by

$$
\mathscr{M}_{n}^{\exp }=\int \frac{\mathrm{d} s}{s^{n+1}} R_{Q}(s)
$$

$R_{Q}$ can naturally be divided into three parts: At lower energies one has the narrow resonances which are the $J / \Psi$ and $\Psi^{\prime}$ for charm the $\Upsilon(n S)(n=1, \ldots, 4)$ in the case of the bottom quark. 

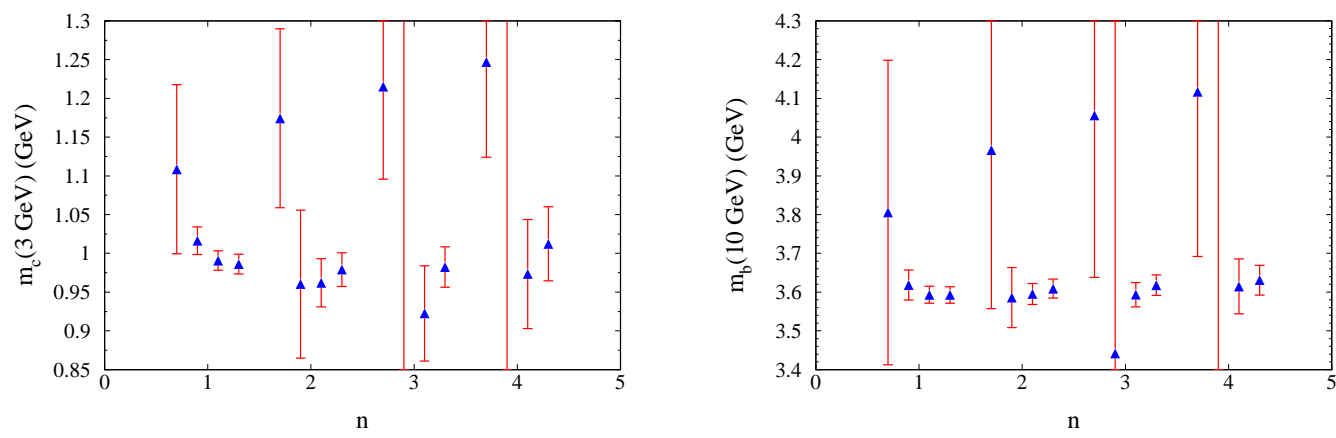

Figure 1: $m_{c}(3 \mathrm{GeV})$ (left) and $m_{b}(10 \mathrm{GeV})$ (right) for $n=1,2,3$ and 4. For each value of $n$ the results from left to right correspond the inclusion of terms of order $\alpha_{s}^{0}, \alpha_{s}^{1}, \alpha_{s}^{2}$ and $\alpha_{s}^{3}$ to the theory-moments.

The second part, usually called threshold region, extends in the case of the charm quark from $3.73 \mathrm{GeV}$ to about $5 \mathrm{GeV}$. In this region the cross section shows a rapid variation and can not be described by perturbation theory. Measurements from the BES collaboration from 2001 [9] and 2006 [21] provide excellent data for $R(s)$ with an uncertainty of about $4 \%$. In order to obtain $R_{c}$ one has to subtract the contribution from the light quarks which is explained in detail in Ref. [15]. Similarly, we refer to Ref. [15] for a discussion of the bottom threshold region.

The third contribution to the experimental moment is provided by the so-called continuum region which for the charm and bottom quark starts above $4.8 \mathrm{GeV}$ and $11.24 \mathrm{GeV}$, respectively. In both cases there is no precise experimental data available. On the other hand, perturbative QCD is supposed to work very well in these energy regions, in particular since $R_{Q}(s)$ is known to order $\alpha_{s}^{2}$ including the full quark mass dependence and to order $\alpha_{s}^{3}$ including quartic mass effects.

Equating the theoretical and experimental moments of Eqs. (3.2) and (3.3), adopting $\mu=$ $3 \mathrm{GeV}(\mu=10 \mathrm{GeV})$ for the charm (bottom) quark and solving for the quark mass leads to the results which are shown in Fig. 1 in graphical form. For detailed numerical results including a dedicated error analysis we refer to Ref. [15]. It is nicely seen that the results for $m_{Q}$ further stabilize when going from three to four loops. At the same time the uncertainty is considerably reduced. Furthermore, the preference for the first three moments is clearly visible. Also the analysis for $n=2$ and $n=3$ leads to small errors, even if we include the uncertainty from the yet uncalculated four-loop contributions (cf. Ref. [15]). We emphasize the remarkable consistency between the three results which we consider as additional confirmation of our approach.

The final result for the $\overline{\mathrm{MS}}$-masses read $m_{c}(3 \mathrm{GeV})=0.986(13) \mathrm{GeV}$ and $m_{b}(10 \mathrm{GeV})=$ $3.609(25) \mathrm{GeV}$. They can be translated into $m_{c}\left(m_{c}\right)=1.286(13) \mathrm{GeV}$ and $m_{b}\left(m_{b}\right)=4.164(25) \mathrm{GeV}$ which currently constitute the most precise mass values. On the theory-side further improvements are possible by evaluating the four-loop moments for $n=2$ and $n=3$. On the experimental side improvements of the electronic widths of the narrow resonances and $R$ measurements in the threshold region and slightly above would be very useful.

\section{Acknowledgments}

We would like to thank Hans Kühn, Christian Strum and Thomas Teubner for the fruitful collaboration on the topics presented in this contribution. This work was supported by the DFG through SFB/TR 9. 


\section{References}

[1] D. Besson et al. [CLEO Collaboration], Phys. Rev. D 76 (2007) 072008 [arXiv:0706.2813 [hep-ex]].

[2] K. G. Chetyrkin, J. H. Kühn and A. Kwiatkowski, Phys. Rept. 277 (1996) 189.

[3] K. G. Chetyrkin, A. H. Hoang, J. H. Kühn, M. Steinhauser and T. Teubner, Eur. Phys. J. C 2 (1998) 137 [arXiv:hep-ph/9711327].

[4] R. V. Harlander and M. Steinhauser, Comput. Phys. Commun. 153 (2003) 244 [arXiv:hep-ph/0212294].

[5] K. G. Chetyrkin, J. H. Kühn and M. Steinhauser, Comput. Phys. Commun. 133 (2000) 43 [arXiv:hep-ph/0004189].

[6] K. G. Chetyrkin, B. A. Kniehl and M. Steinhauser, Nucl. Phys. B 510 (1998) 61 [arXiv:hep-ph/9708255].

[7] M. Steinhauser, Phys. Rept. 364 (2002) 247 [arXiv:hep-ph/0201075].

[8] J. H. Kühn and M. Steinhauser, Nucl. Phys. B 619 (2001) 588 [Erratum-ibid. B 640 (2002) 415] [arXiv:hep-ph/0109084].

[9] J. Z. Bai et al. [BES Collaboration], Phys. Rev. Lett. 88 (2002) 101802 [arXiv:hep-ex/0102003].

[10] A. E. Blinov et al. [MD-1 Collaboration], Z. Phys. C 70 (1996) 31.

[11] R. Ammar et al. [CLEO Collaboration], Phys. Rev. D 57 (1998) 1350 [arXiv:hep-ex/9707018].

[12] W. M. Yao et al. [Particle Data Group], J. Phys. G 33 (2006) 1.

[13] S. Bethke, arXiv:hep-ex/0606035.

[14] V. A. Novikov, L. B. Okun, M. A. Shifman, A. I. Vainshtein, M. B. Voloshin and V. I. Zakharov, Phys. Rept. 41 (1978) 1.

[15] J. H. Kühn, M. Steinhauser and C. Sturm, Nucl. Phys. B 778 (2007) 192 [arXiv:hep-ph/0702103].

[16] K. G. Chetyrkin, J. H. Kühn and M. Steinhauser, Phys. Lett. B 371 (1996) 93 [arXiv:hep-ph/9511430].

[17] K. G. Chetyrkin, J. H. Kühn and M. Steinhauser, Nucl. Phys. B 482 (1996) 213 [arXiv:hep-ph/9606230].

[18] K. G. Chetyrkin, J. H. Kühn and M. Steinhauser, Nucl. Phys. B 505 (1997) 40 [arXiv:hep-ph/9705254].

[19] K. G. Chetyrkin, J. H. Kühn and C. Sturm, Eur. Phys. J. C 48 (2006) 107 [arXiv:hep-ph/0604234].

[20] R. Boughezal, M. Czakon and T. Schutzmeier, Phys. Rev. D 74 (2006) 074006 [arXiv:hep-ph/0605023].

[21] M. Ablikim et al. [BES Collaboration], arXiv:hep-ex/0612054. 